\title{
Policondritis recurrente, una rara causa de valvulopatía: revisión de las publicaciones médicas
}

\author{
Relapsing polychondritis, a rare cause of valvulopathy: a review of the medical literature \\ Eduardo Bahena-López ${ }^{1 *}$ y Jorge Loya-Centurión ${ }^{2}$ \\ ${ }^{1}$ Departamento de Hemodinámica; ${ }^{2}$ Departamento de Cardiología Clínica. Instituto Nacional de Cardiología "Ignacio Chávez", Ciudad de México, \\ México
}

\section{Resumen}

La policondritis recurrente (PR) es una enfermedad inflamatoria que afecta a estructuras cartilaginosas, predominantemente las que se encuentran en nariz, pabellones auriculares y vías respiratorias. Las manifestaciones cardiovasculares son poco comunes; sin embargo, son la segunda causa de mortalidad en pacientes con PR. Unas de las estructuras afectadas casi siempre en la PR son las estructuras valvulares y la valvulopatía más común es la insuficiencia aórtica (IA). A continuación se presenta el caso de una paciente sin diagnóstico previo de PR a quien se refirió a este instituto por insuficiencia cardíaca secundaria a IA, atribuida en un principio a endocarditis.

Palabras clave: Policondritis recurrente. Enfermedad valvular. Insuficiencia aórtica. México.

\begin{abstract}
Relapsing polychondritis is an inflammatory disease that involves cartilaginous structures predominantly in the nose, ears and respiratory tract. Cardiovascular involvement is not common. Despite this, they are the second cause of death in patients with relapsing polychondritis. The structures usually affected by this disease are the heart valves, with regurgitation being the most common valvulopathy. We present the case of a patient without previous diagnosis of relapsing polychondritis who was referred to our Institute with heart failure secondary to aortic regurgitation, initially attributed to endocarditis.
\end{abstract}

Key words: Relapsing plychondritis. Valvular disease. Aortic regurgitation. Mexico.

\section{Introducción}

Mujer de 38 años con antecedente de colitis ulcerativa crónica inespecífica (CUCl) bajo tratamiento con mesalazina. Presentó cuadro caracterizado por astenia, adinamia, fiebre de $40^{\circ} \mathrm{C}$ junto con escalofrío, además de evacuaciones líquidas con trazas de sagrado, tenesmo e incontinencia. Fue hospitalizada en otra institución en donde se detectó un soplo cardíaco. El ecocardiograma transtorácico (ETT) mostró IA grave. Debido a estos hallazgos se le trasladó al Instituto Nacional de Cardiología de México. A su ingreso se encontraron datos clínicos de IA grave, signo de Landolfi positivo, pulso de Corrigan, precordio con
Correspondencia:

*Eduardo Bahena-López

E-mail: eduardobahena @ comunidad.unam.mx DOI: 10.24875/ACM.19000245
Disponible en internet: 29-01-2020 Arch Cardiol Mex. 2020;90(2):189-192 www.archivoscardiologia.com BY-NC-ND (http://creativecommons.org/licenses/by-nc-nd/4.0/). 
un soplo aspirativo, holodiastólico en foco aórtico accesorio irradiado al ápex, extremidades con pulsos periféricos amplios, signo de Lyan y Quincke positivos, además de pabellón auricular izquierdo con aumento de tamaño, edema, eritema y dolor a la palpación.

Se realizó ETT que reveló ventrículo izquierdo dilatado con fracción de expulsión de $50 \%$, ventrículo derecho con función sistólica conservada, válvula aórtica trivalva con prolapso de valva coronariana izquierda y dilatación de la raíz aórtica que producía IA grave (Fig. 1A y B). Se completó abordaje con ecocardiograma transesofágico que mostró baja probabilidad de endocarditis y se corroboraron los hallazgos descritos con anterioridad en el ETT (Fig. 1C y D).

Debido al antecedente de CUCl se la valoró en el Servicio de Gastroenterología; se realizó colonoscopia que identificó pancolitis secundaria a $\mathrm{CUCl}$. En virtud de la aparente condritis auricular, se la valoró también en el Servicio de Reumatología por sospecha de PR. Se integró diagnóstico de PR por la presencia de tres criterios de McAdam: condritis auricular, condritis nasal y daño audiovestibular (hipoacusia unilateral izquierda corroborada mediante audiometría). Se inició tratamiento con hidrocortisona y metotrexato. Debido a la IA se propuso operación de cambio valvular aórtico, pero la paciente rechazó el procedimiento. La paciente egresó con tratamiento a base de prednisona y metotrexato y siguió bajo seguimiento por consulta externa. En consulta de seguimiento dos meses después de su egreso se advirtió mejoría del proceso inflamatorio auricular, así como mejoría de síntomas cardiovasculares (clase funcional I de la NYHA).

\section{Discusión}

La PR es una enfermedad inflamatoria sistémica que se caracteriza por episodios repetitivos de inflamación de estructuras cartilaginosas y tejidos con alta concentración de proteoglucanos (ojos, corazón, vasos sanguíneos). Su incidencia anual es de 3.5 casos por cada millón de habitantes, con un punto máximo de incidencia entre los 30 y 55 años ${ }^{1}$. La causa se desconoce, aunque parece haber cierta susceptibilidad genética. Se ha descrito una relación entre la PR y el complejo mayor de histocompatibilidad tipo II, de modo específico con el HLA-DR4. La inmunidad humoral juega un papel importante en la fisiopatología mediante la producción de autoanticuerpos, sobre todo contra la colágena tipo II. También se identifica producción de autoanticuerpos contra la colágena tipos IX y XI, así como matrilina 1 y proteínas de matriz extracelular presentes en el cartílago ${ }^{2}$. Otro mecanismo fisiopatológico relevante es la elevación de citocinas proinflamatorias que intervienen en la activación de macrófagos, monocitos y leucocitos en pacientes con PR, lo cual sugiere afectación de la inmunidad celu$\operatorname{lar}^{3}$, la cual también parece estar dirigida en contra de la colágena tipo $\mathrm{II}^{4}$.

Las manifestaciones clínicas más frecuentes de la PR son la condritis auricular (36-81\%) y nasal (29$54 \%$ ), la primera la manifestación más prevalente de forma inicial. Existen asimismo manifestaciones cardiovasculares, renales y neurológicas, las cuales son menos comunes ${ }^{1}$. Las manifestaciones cardiovasculares incluyen aortitis, aneurismas aórticos, valvulopatías (insuficiencia aórtica o mitral), alteraciones del sistema de conducción, pericarditis, entre otras ${ }^{5,6}$.

La mortalidad relacionada con complicaciones cardiovasculares es la segunda causa de muerte después de las complicaciones laringotraqueales. Dentro de estas complicaciones destaca la afección aórtica, que se ha documentado en $5 \%$ a $10 \%$ de las personas con PR. Las lesiones aórticas pueden ser asintomáticas en etapas tempranas y parece existir cierta selectividad por la aorta torácica (82-92\%) y hasta un tercio de los pacientes presenta afección de aorta torácica y abdominal. Las anomalías aórticas más frecuentes descritas en estos pacientes son aneurismas aórticos aislados y aortitis. Es posible que la presencia de aortitis crónica sea parte de la fisiopatología del desarrollo de los aneurismas aórticos ${ }^{5}$. Con base en lo anterior, las manifestaciones clínicas cardiovasculares más comunes se atribuyen a la regurgitación aórtica secundaria a dilatación de la raíz aórtica ${ }^{2,7}$.

El diagnóstico de PR se establece mediante los criterios de McAdam; se requiere un total de tres criterios para determinar el diagnóstico. Estos criterios incluyen condritis auricular, poliartritis no erosiva, condritis nasal, inflamación ocular (conjuntivitis, queratitis, uveítis, escleritis), condritis del tracto respiratorio (laringe o tráquea) y alteraciones cocleares o vestibulares (pérdida de audición, acúfenos, vértigo) ${ }^{8}$.

No existen estudios de laboratorio específicos para el diagnóstico de la policondritis. Cuando existe duda diagnóstica, la biopsia de pabellón auricular es útil para corroborar la sospecha diagnóstica.

El uso de TEP-TC con FDG tiene utilidad para el diagnóstico cuando el cuadro carece de manifestaciones clínicas típicas y se identifica un aumento de la captación en sitios afectados, incluida la aorta ${ }^{2}$. Además, sirve en el seguimiento para evaluar la respuesta del tratamiento inmunosupresor?. 


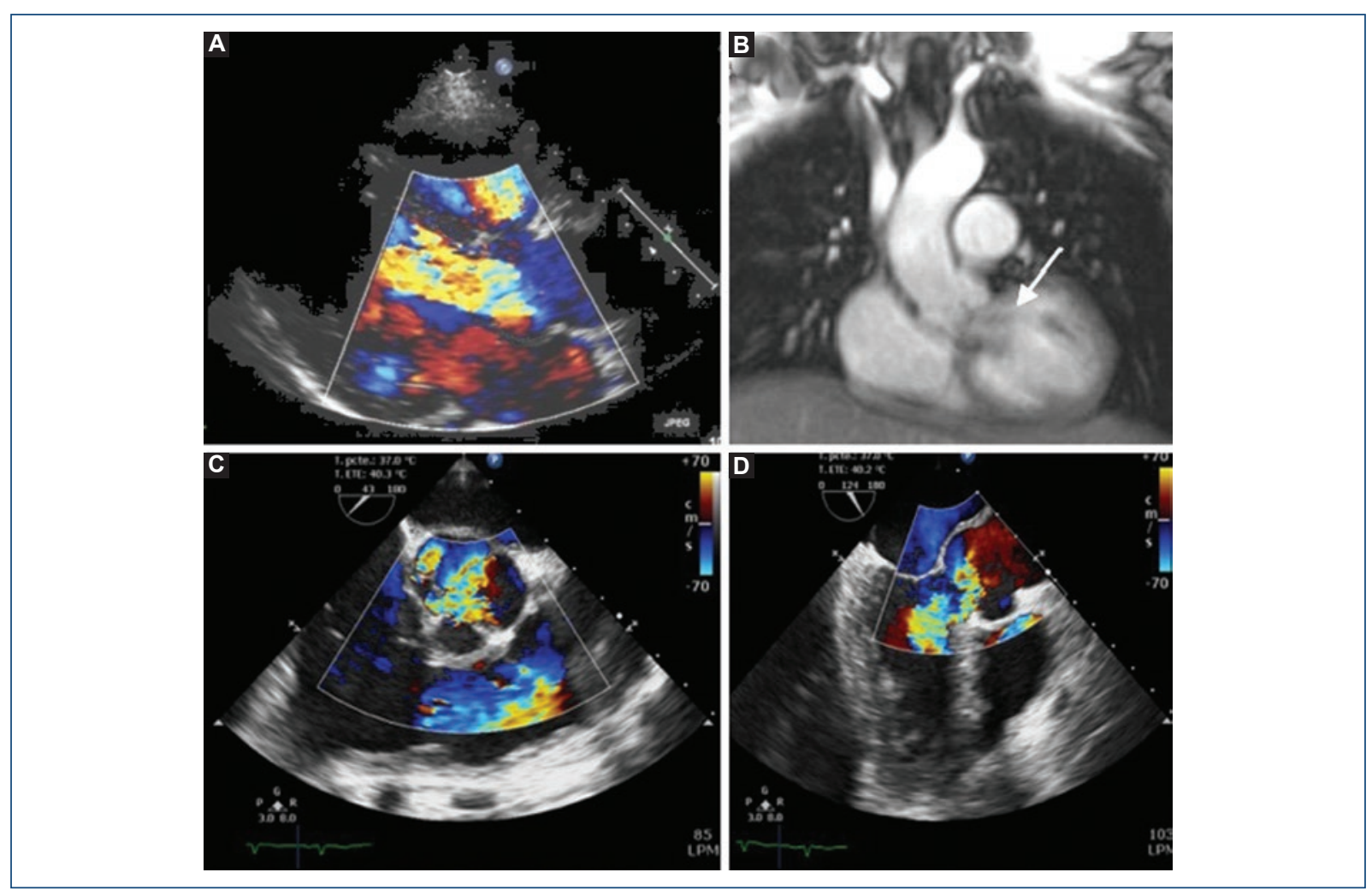

Figura 1. A: Ecocardiograma transtorácico: eje paraesternal largo con técnica Doppler a color que muestra insuficiencia cardíaca. B: Imagen de resonancia magnética que delinea flujo regurgitante aórtico (flecha). C y D: Imágenes con Doppler a color de ECOTE que muestran flujo de insuficiencia aórtica.

Para el tratamiento de la PR suelen emplearse medicamentos inmunosupresores e inmunomoduladores, pero ninguno de ellos ha mostrado modificar la evolución natural de la enfermedad. Factores como los órganos afectados, la gravedad y la duración de la actividad deben tomarse en cuenta para tomar decisiones terapéuticas. El tratamiento con glucocorticoides es la piedra angular del tratamiento de esta enfermedad. En casos en los que la enfermedad es moderada o grave, con riesgo de daño a órganos afectados, está indicado el uso de tratamiento combinado, con adición de ciclofosfamida, azatioprina o metotrexato al tratamiento con glucocorticoides. Si a pesar de esto no se obtiene respuesta adecuada al tratamiento, se pueden prescribir agentes biológicos como los antagonistas del factor de necrosis tumoral alfa: infliximab, etanercept y adalimumab. Otros compuestos biológicos administrados en el tratamiento de la PR como tocilizumab (anticuerpo anti-IL-6) y abatacept (inhibidor de activación de células T) han mostrado ser efectivos para el tratamiento de PR en algunas series de casos.

Las indicaciones para el tratamiento quirúrgico y el tiempo ideal para practicar una intervención son controversiales dado que el tratamiento quirúrgico conlleva múltiples complicaciones postquirúrgicas por el alto riesgo de dehiscencia protésica temprana y recurrencia del aneurisma debido a la friabilidad del tejido causada por la inflamación recurrente, incluso en pacientes con enfermedad leve ${ }^{5}$. Debido a esto, algunos autores recomiendan reemplazar la aorta ascendente siempre que se realice cambio valvular aórtico en pacientes con $\mathrm{PR}^{10}$.

Durante el seguimiento periódico de pacientes PR debe vigilarse el compromiso cardiovascular de forma dirigida, dado que éste no sólo ocurre durante la fase inflamatoria aguda de la enfermedad, sino que puede presentarse aun en pacientes asintomáticos ${ }^{5}$.

\section{Conclusión}

Las manifestaciones cardiovasculares de la PR son la segunda causa de muerte en pacientes con esta enfermedad. La insuficiencia aórtica es la valvulopatía más común en PR y el tratamiento consiste en inmunosupresores para disminuir la inflamación y realizar una operación de cambio valvular. El tratamiento 
quirúrgico implica múltiples complicaciones debido a la friabilidad del tejido causada por la inflamación recurrente.

\section{Financiación}

Los autores no recibieron ningún tipo de financiación de ningún organismo.

\section{Conflicto de intereses}

La presente investigación no ha recibido ninguna beca específica de agencias de los sectores público, comercial o sin ánimo de lucro.

\section{Responsabilidades éticas}

Protección de personas y animales. Los autores declaran que para esta investigación no se han realizado experimentos en seres humanos ni en animales.

Confidencialidad de los datos. Los autores declaran que han seguido los protocolos de su centro de trabajo sobre la publicación de datos de pacientes.
Derecho a la privacidad y consentimiento informado. Los autores han obtenido el consentimiento informado de los pacientes y/o sujetos refereridos en el artículo.

\section{Bibliografía}

1. Pallo PAO, Levy-Neto M, Pereira RMR, Shinjo SK. Relapsing polychondritis: prevalence of cardiovascular diseases and its risk factors, and general disease features according to gender. Rev Bras ReumatolEngl Ed 2017:57(4):338-45.

2. Mathian A, Miyara M, Cohen-Aubart F, Haroche J, Hie M, Pha M, et al. Relapsing polychondritis: a 2016 update on clinical features, diagnostic tools, treatment and biological drug use. Best Pract Res ClinRheumatol 2016;30(2):316-33

3. Stabler T, Piette JC, Chevalier X, Marini-Portugal A, Kraus VB. Serum cytocine profiles in relapsing polychondritis suggest monocyte/macrophage activation. Arthritis Rheum 2004:50(11):3663-7.

4. Buckner JH, Van Landeghen M, Kwok WW, Tsarknaridis L. Identification of type II collagen peptide $261-273$-specific $T$ cell clones in a patient with relapsing polychondritis. Arthritis Rheum 2002;46(1):238-44.

5. Del Rosso A, Petix NR, Pratesi M, Bini A. Cardiovascular involvement in relapsing polychondritis. Semin Arthritis Rheum 1997;26:840-844.

6. Kingdon J, Roscamp J, Sangle S, D'Cruz D. Relapsing polychondritis: a clinical review for rheumatologist. Rheumatology (Oxford) 2018:57(9): 1525-1532.

7. Le Besnerais M, Arnaud L, Boutémy J, Bienvenu B, Lévesque $H$, Amoura $Z$, et al. Aortic involvement in relapsing polychondritis. Joint Bone Spine 2018;85(3):345-51

8. McAdam LP, O'Hanlan MA, Bluestone R, Pearson CM. Relapsing polychondritis: prospective study of 23 patients and a review of the literature. Medicine (Baltimore) 1976

9. Lei W, Zeng H, Zeng DX, Zhang B, Zhu YH, Jiang JH, et al. FDG PETCT as a powerful tool for diagnosing and monitoring treatment outcomes of relapsing polychondritis. Eur J Nucl Med Mol Imaging 2018;45(4):669-70.

10. Lang-Lazdunski $L$, Pansard $Y$, Hvass $U$. Aortic valve replacement in relapsing polychondritis. J Thorac Cardiovasc Surg 1997;114:131-2. 